\title{
Selection of Cold Chain Logistics Distribution Center Location Based on Improved Genetic Algorithm
}

\author{
Jianchang Lu and Yaxin Zhao \\ Department of Economic Management, North China Electric Power University, Baoding 071003, Hebei Province, China
}

\begin{abstract}
With the rapid development of food refrigeration and freezing technology, food cryogenic storage and vehicle transportation scheduling technology, the cold chain logistics industry has entered a period of rapid development. According to the problem of urban cold chain distribution route, based on the vehicle distribution model with time window, the minimum cost of transportation, cost of energy, cost of goods, penalty cost is the objective function, and the urban cold chain logistics distribution path is established. Optimized mathematical model. According to the actual case, the analysis of the cold chain distribution model is carried out by using the analytical genetic algorithm, and the optimal combination of the distribution paths with the lowest total cost is obtained, which has certain reference significance for the urban cold chain logistics distribution route problem.
\end{abstract}

\section{Introduction}

With the development of social economy and the continuous improvement of people's living standards, people's consumption patterns have also changed, and more and more attention has been paid to the quality and safety of food. However, frequent food safety incidents have caused all parties in the community to pay close attention to food safety issues. Due to the late start of China's cold chain logistics industry, the overall infrastructure in the industry is relatively weak. In order to meet the growing consumer demand, while developing the cold chain logistics, we are facing a common problem - the cold chain logistics distribution center. How to make a reasonable and effective site selection. In the logistics network, the distribution center connects the supply point and the demand point, which is the bridge between the two and plays a decisive role in the cold chain logistics system[1]. The rational location of the logistics distribution center can save costs, speed up the circulation of goods, and increase the income of logistics enterprises. Therefore, the location problem of the cold chain logistics distribution center is a very important issue for the optimization of the entire cold chain logistics system. At the same time, the key to the success of cold chain distribution is low temperature. In order to ensure low temperature, the two links of transportation and storage become the core. As a combination point, the role of the distribution center is particularly prominent, and the location of the cold chain distribution center is reasonable. It has become a top priority[2-4].

\section{Problem description and mathematical model}

\subsection{Problem description}

For the location problem of the distribution center, the first factor to consider is the shortest path and the lowest cost. For the location of the distribution center of cold chain logistics, due to the special nature of the cold chain food, the transportation distance cannot be too long, the storage conditions and each Transportation links must be carried out in strict accordance with strict standards. Therefore, this puts higher requirements on the distribution of cold chain logistics, which is obviously different from the location problem of ordinary distribution centers.

Description of the problem: The existing supply point $i$ is supplied to a number of distribution centers $j$, and is distributed to the demand point $\mathrm{m}$ after being processed by the distribution center. Assumption: the delivery distance must not be greater than the longest distance traveled by the vehicle; the rated load of the vehicle is not limited; the time spent loading and unloading the required delivery vehicle is not considered; the upper and lower thresholds of the delivery lead time accepted by the customer at the demand point It is known that goods are faster in the distribution center and there is no long-term storage phenomenon[2,5,7].

Definition: $P_{i}$ is the maximum supply of supply point $\mathrm{i} ; \mathrm{C}_{1}(\mathrm{i})$ is the fixed operating cost of supply point $\mathrm{i}$; $\mathrm{J}$ is the number of cold chain distribution centers; $\mathrm{N}$ is the maximum number of construction centers in the 
distribution center; $\mathrm{C}_{2}(\mathrm{i})$ fixed cost of distribution center $\mathrm{j}$; $\mathrm{Vc}(\mathrm{jm})$ is the processing and processing cost (variable cost) of the goods in the distribution center; $M$ is the number of demand points; $D_{m}$ is the demand; $C_{a}$, ij and $C_{b}$, $\mathrm{jm}$ are unit freight rates respectively; $\mathrm{X}_{\mathrm{a}, \mathrm{ij}}$ and $\mathrm{X}_{\mathrm{b}, \mathrm{jm}}$ are

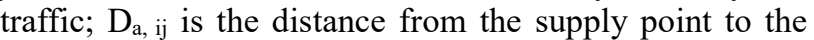
distribution center; $D_{b}, j m$ is the distance from the distribution center to the customer $m ; Y_{b}(i, j)$ is a variable of 0,1 . If the distribution center $\mathbf{J}$ is actually operating, $\mathrm{y}_{\mathrm{b}}(\mathrm{i}, \mathrm{j})=1$, otherwise 0 .

\subsection{Establishing a mathematical model}

First, the goal of establishing a mathematical model is to minimize the total cost. The total cost includes the transportation and distribution cost, the fixed operating cost of the supply point and the distribution center, and the variable cost of the goods circulation processing of the distribution center. The following mathematical model is established:

$$
\begin{aligned}
& \min C=\sum_{i=1}^{I} \sum_{j=1}^{J} c_{a, i j} x_{a, i j} d_{a, i j}+\sum_{j=1}^{J} \sum_{m=1}^{M} c_{b, j m} x_{b, j m} d_{b, j m} \\
& +\sum\left(\sum_{i=1}^{I} \sum_{j=1}^{J} y_{b}(i, j) c_{1}(i)+y_{b}(j, m) c_{2}(j)\right) \\
& +\sum_{j=1}^{J} \sum_{m=1}^{M} v_{c}(j m) x_{b, j m}
\end{aligned}
$$

Restrictions:

$$
\begin{gathered}
\sum_{i=1}^{I} \sum_{j=1}^{J} x_{a, i j} y_{b}(i, j) \leq \sum_{i=1}^{I} p \\
\sum_{j=1}^{J} x_{b, j m} \geq D_{m} \\
\sum_{j=1}^{J} \sum_{m=1}^{M} x_{a, j m} y_{b}(j, m) \leq \sum_{m=1}^{M} D_{m} \\
y_{b}(j, m)=0, \text { or } 1 . \forall m, j=1,2, \ldots, J \\
\sum_{j=1}^{J} y_{b}(j, m) \leq N \\
\sum_{i=1}^{I} \sum_{j=1}^{J} x_{a, i j} \geq 0, \sum_{j=1}^{J} \sum_{m=1}^{M} x_{b}, j m \geq 0 \\
\sum_{i=1}^{I} \sum_{j=1}^{J} x_{i j m}=0
\end{gathered}
$$

In the above model: Equation (1) is the objective function, that is, the total cost is the smallest; Equation (2) is the supply quantity constraint of each supply point, indicating that the actual supply quantity of each supply point $i$ cannot be greater than its actual capacity; Equation (3) indicates that the demand for each demand point must be met; Equation (4) indicates that the quantity of goods actually delivered by the distribution center cannot be greater than the total demand of the customer, and logical variables must be considered; Equation (5) indicates that the cold chain logistics distribution center is equal to 1 if it is selected for operation, otherwise 0; Equation (6) indicates that the total number of distribution centers cannot be greater than N; Equation (7) indicates that the traffic is a non-negative constraint; Equation (8) ensures no vehicle operation between cold chain distribution centers.

Due to the distribution problem of cold chain logistics, it is also necessary to consider the time window constraints of customer demand. That is, each delivery task must be completed within the time period specified by the customer, otherwise the corresponding cost will be incurred. Let ta be the time when the vehicle arrives at the demanding customer $\mathrm{a}(\mathrm{a} \in \mathrm{m})$, and the time period allowed for the delivery task is $[t \min , t \max ]$ among them: $t$ min is the earliest allowed arrival time; $t$ max is the latest allowed time of arrival. If the vehicle arrives before $t$ min, There is a loss of opportunity cost due to the waiting of the vehicle. Recorded as

$$
l_{1}=\delta\left(t_{\min }-t_{a}\right)^{2}
$$

If the vehicle arrives before $t$ max , the corresponding delay penalty is applied. Recorded as

$$
l_{2}=\rho\left(t_{a}-t_{\max }\right)^{2}
$$

Therefore, the penalty cost in the location of the cold chain distribution center is:

$$
C_{p}=\delta \sum_{j=1}^{m}\left(\max \left(t_{\min }-t_{a}, 0\right)\right)^{2}+\rho \sum_{j=1}^{m}\left(\max \left(t_{a}-t_{\max }, 0\right)\right)^{2}
$$

In the formula, $\delta, \quad \rho$ is a constant term, determined according to the contractual agreement with the customer; $t_{\text {min }} \leq t_{a} \leq t_{\text {max }}$

Moreover, because the cold chain products are characterized by freshness, perishability and timeliness, the market price of the products will fall with the passage of time. Therefore, in the cold chain logistics transportation, the product quality loss rate $\mu \in(0,1)$ is often used to characterize the customer service level. Assume that the residual quality rate of cold chain products generated from the production supply point $i$ to the cold chain logistics distribution center $j$ is $\mu_{\mathrm{j} m} \in(0,1)$.Based on the 3T theory of frozen products, the loss rate of cold chain products is

$$
\mu=k \sqrt{t / t_{G P}}
$$

In the above formula, $\mathrm{t}$ is the transportation time of the goods, $t_{G P}$ is the product shelf life, so the mathematical model expression of the customer service level is:

$$
\min \mu=\tau \sqrt{\frac{1}{t_{G P}}\left(\sum_{i=1}^{I} \sum_{j=1}^{J} \sum_{m=1}^{K} \frac{d_{i j m}}{k_{i j m}}+\frac{1}{v_{c}^{2}(j m)}\right)}
$$

Restrictions:

$$
\sum_{i=1}^{I} \sum_{j=1}^{J} \sum_{m=1}^{M}\left(\frac{d_{a, i j}}{k_{a, i j}}+\frac{d_{b, j m}}{k_{b}, j m}\right) \leq t_{c l}
$$


Therefore, the objective function of the location optimization model of the cold chain logistics distribution center is to consider the transportation and distribution costs, the fixed cost and operating cost of the supply point and the distribution center, the variable cost of the goods circulation processing of the distribution center, and the time window constraint considering the demand. The sum of the penalty cost and the total cost of considering the loss rate of the goods:

$$
\begin{aligned}
& \min C=\min \left[\sum_{i=1}^{I} \sum_{j=1}^{J} c_{a, i j} x_{a, i j} d_{a, i j}\right. \\
& +\left(\sum_{j=1}^{J} \sum_{m=1}^{M} c_{b, j m} x_{b, j m} d_{b, j m}+\sum_{i=1}^{I} \sum_{j=1}^{J} y_{b}(i, j) c_{1}(i)\right. \\
& \left.+y_{b}(j, m) c_{2}(j)\right)+\sum_{j=1}^{J} \sum_{m=1}^{M} v_{c}(j m) x_{b, j m} \\
& +\tau \sqrt{\frac{1}{t_{G P}}\left(\sum_{i=1}^{I} \sum_{j=1}^{J} \sum_{m=1}^{M} \frac{d_{i j m}}{k_{i j m}}+\frac{1}{v_{c}^{2}(j m)}\right)} \\
& +\left(\delta \sum_{j=1}^{J}\left(\max \left(t_{\min }-t_{a}, 0\right)\right)^{2}\right. \\
& \left.+\rho \sum_{j=1}^{J}\left(\max \left(t_{a}-t_{\max ,} 0\right)\right)^{2}\right]
\end{aligned}
$$

\subsection{Improved genetic algorithm design}

The location of the cold chain distribution center is a complex combinatorial optimization problem, so the corresponding heuristic algorithm must be designed for research. The genetic algorithm uses the genetic mechanism to continuously update the population, and has strong parallel and global search ability, but the genetic algorithm has a slower evolution rate and is easy to fall into local optimum. The Tabu algorithm (TA) has an adaptive storage mode memory function, which can effectively avoid local convergence, but the global search ability is poor. Therefore, the idea of TA search algorithm can be integrated in genetic algorithm to increase the global optimization ability of genetic algorithm. It is embodied in the following two aspects:(1)Improve the crossover operator. The validity of the crossover operator directly affects the efficiency of the genetic algorithm. In the algorithm design, the greedy algorithm is used to adjust the crossover probability, and the evolution process is dynamically adjusted according to the fitness function. (2)Import the TAsearch process. Mutation operations generate new individuals through information exchange and combinatorial genes[4,6]. The TA search algorithm is applied to the mutation operation, and the taboo table with memory storage characteristics can avoid falling into local optimum.

\subsubsection{Genetic algorithm steps}

The first is the choice strategy. Use the usual "roulette" proportional selection strategy. Suppose the population size is $N_{P}, X_{\lambda}^{(\sigma)}$ is the $\sigma$ individual of $X^{(\sigma)}$ in the $\lambda$ generation population, ${ }^{E_{\lambda}^{(\sigma)}}$ is the fitness of $X_{\lambda}^{(\sigma)}$,
Calculation $P_{\lambda}=F_{\lambda}^{(\sigma)} \sum_{\lambda=1}^{N} F_{\lambda}^{(\sigma)}$ In the above formula, $P_{\lambda}$ is the probability of selection; ${ }^{F_{\lambda}^{(\sigma)}}$ is the fitness. Let $P_{0}$ $=0$. Randomly generate a number $\phi$ that is uniformly distributed from 0 to 1.If $\sum_{i=0}^{n-1} P_{\lambda} \in \phi \leq \sum_{\lambda=0}^{i} P_{i}$ Choose $x_{2}^{(\sigma)}$ as the next generation's father, Repeat until ${ }^{N}{ }_{p}$ parent size is selected. The next Cross operation. The greedy algorithm is used to adaptively improve the cross mutation operator. The improved mutation rate is dynamic. The definition of the crossover probability is: $P_{c}=1 /\left[1+\exp \left(K_{1} \Delta\right)\right] \quad(16)$ In the above formula, $\Delta=f_{\text {avg }}-f_{\max }, f_{\text {avg }}$ is the average fitness value, $f_{\text {max }}$ is the maximum fitness value; $\mathrm{K}_{1}$ is a constant coefficient. Create a random number $\varepsilon, \varepsilon \in[0,1]$, if $\sigma<P_{c}$, then select the given gene to cross. The two generations of children are randomly generated as:

$$
\begin{aligned}
& v_{1}=\left(b_{1}, b_{2}, \ldots, b_{m}, b_{p o s 1}, b_{p o s 1+1}, \ldots, b_{p o s 1+m}, b_{p o s},\right. \\
& b_{p o s 2+1}, \ldots, b_{p o s 2+m}, \ldots, b_{p o s m}, b_{p o s m+1}, \ldots, b_{p o s m+m}, b_{m} \\
& v_{2}=\left(c_{1}, c_{2}, \ldots, c_{m}, c_{p o s 1}, c_{p o s 1+1}, \ldots, c_{p o s 1+m}, c_{p o s 2},\right. \\
& c_{p o s 2+1}, \ldots, c_{p o s 2+m}, \ldots, c_{p o s m}, c_{p o s m+1}, \ldots, c_{p o s m+m}, c_{m}
\end{aligned}
$$

Where $b_{1}, b_{2}, \ldots \quad$ is the first, second, ... chromosome of the first group of progeny; ${ }_{\text {pos } 1}, b_{\text {pos } 1+1}, \ldots$ is the first, second, ... chromosome of the first set of progeny variations; $c_{1}, c_{2}, \ldots$ is the first, second, ... chromosome of the second group of progeny; $c_{\text {pos } 1}, c_{p o s}{ }_{1+1}, \ldots$ is the first, second, ... chromosome of the second set of progeny variations; and so on. Finally, Mutation operation. [8,9] The mutation operator refers to replacing the gene values at certain loci in the individual chromosome coding string with other alleles of the locus, thereby forming a new individual and improving the local search ability.

\section{Example analysis}

Suppose there are 2 supply bases, and the users are distributed in 8 areas. The initial planning is to set up 3 distribution centers, namely $\mathrm{P}=3$, and there are 5 distribution center candidates D1, D2, D3, D4 and D5.It is known that the unit variable cost of the distribution center is $8,12,11,10,10$ in order. $\theta=1$,Fixed cost investment is 120 ; The specific data of the supply capacity and user demand of each supply base, the supply base to the distribution center and the distribution center to the user's unit freight are shown in Table 1 and Table 2[11].

Table 1. Unit freight and supply capacity from each supply base to the distribution center

\begin{tabular}{llllll}
\hline \multirow{2}{*}{ Supply } & \multicolumn{4}{c}{ Unit shipping to the distribution center } \\
\cline { 2 - 6 } capacity & D1 & D2 & D3 & D4 & D5 \\
\hline 40 & 7 & 7 & 8 & 12 & 11 \\
50 & 14 & 12 & 9 & 6 & 8 \\
\hline
\end{tabular}


Table 2. The unit freight and user demand of each distribution center to the user

\begin{tabular}{llllllll}
\hline \multirow{2}{*}{ Delivery } & \multirow{2}{*}{ Maximum } & \multicolumn{7}{c}{ Unit freight to demand city } \\
\cline { 3 - 7 } center & capacity & U1 & U2 & U3 & U4 & U5 & U6 \\
\hline D1 & 30 & 5 & 11 & 3 & 8 & 5 & 10 \\
D2 & 20 & 14 & 16 & 8 & 9 & 4 & 7 \\
D3 & 35 & 10 & 11 & 3 & 5 & 1 & 5 \\
D4 & 35 & 15 & 13 & 9 & 6 & 7 & 2 \\
D5 & 25 & 9 & 7 & 3 & 2 & 6 & 5 \\
Demand & -- & 10 & 10 & 10 & 15 & 5 & 15 \\
\hline
\end{tabular}

Based on the Mat lab 7.0 as a platform, based on the genetic algorithm toolbox developed by Sheffield University, the genetic operator based and genetic function are designed. The parameters are set as follows: Maximum iteration number max_gen $=300$; population size pop_size $=50$; Hybrid probabilit $P_{\mathrm{c}}=0.5$; Mutation probability $P_{m}=0.1$ D1, D4 and D5 were selected. The optimal distribution scheme is shown in Tables 3 and 4. The total cost is 2220 .

Table 3. Transportation plan from supply base to distribution center

\begin{tabular}{lllll}
\hline \multirow{2}{*}{ Supply base } & Supply & \multicolumn{3}{c}{ Delivery center } \\
\cline { 3 - 5 } & capacity & D1 & D4 & D5 \\
\hline S1 & 40 & 30 & -- & 10 \\
S2 & 50 & -- & 35 & 15 \\
Maximum & -- & 30 & 35 & 25 \\
capacity & & & & \\
\hline
\end{tabular}

Table 4. Distribution plan from distribution center to user

\begin{tabular}{llllllll}
\hline \multirow{2}{*}{ Delivery } & \multirow{2}{*}{ Maximum } & \multicolumn{8}{c}{ Client } \\
\cline { 3 - 7 } center & capacity & U1 & U2 & U3 & U4 & U5 & U6 \\
\hline D1 & 30 & 10 & - & 10 & - & 5 & - \\
D4 & 35 & - & - & - & - & - & 15 \\
D5 & 25 & - & 10 & - & 15 & - & - \\
Demand & - & 10 & 10 & 10 & 15 & 5 & 15 \\
\hline
\end{tabular}

\section{Conclusion}

The location of the cold chain logistics distribution center involves not only the costs of construction, operation and distribution, but also the level of customer service and quality of goods. Therefore, an optimization model for the location of cold chain logistics distribution center considering factors such as cost, time window constraint, cargo damage rate and customer service level is constructed, which has practical application value. Because this model is a complex system optimization problem, Therefore, a hybrid genetic algorithm based on fusion genetic algorithm and tabu search algorithm and greedy algorithm to improve crossover operator is designed. This improved hybrid genetic algorithm embeds the tabu search algorithm into the mutation operation process in a single genetic algorithm, overcomes the problem of premature convergence and convergence of a single heuristic algorithm, and uses the greedy algorithm to adaptively improve the crossover operator to further improve the iteration. Efficiency provides a new solution to the problem of complex system optimization, with certain theoretical and practical value.

\section{References}

1. Fang Wenchao. Study on Location Selection of Distribution Center Based on Genetic Algorithm[J]. Beijing City Studies Journal of the Academy, 2015, (1)

2. Zhao Wanhua. Study on the Evaluation Method of Location Selection of Regional Logistics Distribution Center[D]. Wuhan University, 2011

3. Yuan Qun. Location Optimization of Cold Chain Logistics Distribution Center Based on Improved Hybrid Genetic Algorithm[J]. Journal of Shanghai Jiaotong University, 2016(11):1795-1800

4. Huang Zunjuan. Research on Optimization of Urban Cold Chain Logistics Distribution Route Based on Genetic Algorithm[J]Economy and management,2017(8):97-101

5. Yang Danting. Cold chain logistics distribution path optimization[D].Dalian Maritime University,2014

6. WU Qinggang. Research on the Status Quo and Countermeasures of China's Cold Chain Logistics Development[]]China's circulation economy, 2011, (2):24-28

7. Li Yuhui, Zhen Guohua, et al. Optimization of Multi-temperature Co-allocation Cost of Cool Storage Cooling Products Based on Genetic Algorithm[J]. Journal of Railway Science and Engineering, 2017(4):879-884

8. Zhang Pengcheng. Research on Cold Chain Logistics Path Optimization Based on Improved Genetic Algorithm[D]. Anhui University of Science and Technology,2016

9. Dong Jie. Research on Optimization of Urban Cold Chain Logistics Distribution Route under Green Perspective[D]. Dalian University of Technology, 2017

10. Li Shuaishuai, Zhang Zheng, et al. Research on Optimization of Cold Chain Logistics Transportation Mode under Axial-spoke 
Multimodal Transport Network[]]. Logistics technology,2016(7):78-81

11. Yu Lei, Shi Feng, Wang Hui, et al. 30 Case Analysis of MATLAB Intelligent Algorithm [M].
Beijing University of Aeronautics and Astronautics Press, 2015 